\title{
Parental Knowledge/Monitoring and Depressive Symptoms During Adolescence: Protective Factor or Spurious Association?
}

\author{
William E. Pelham III ${ }^{1}$ - Susan F. Tapert ${ }^{1}$ - Marybel R. Gonzalez ${ }^{1}$. Mathieu Guillaume ${ }^{2}$. Anthony Steven Dick ${ }^{3}$. \\ Chandni S. Sheth ${ }^{4}$. Fiona C. Baker ${ }^{5}$. Arielle Baskin-Sommers ${ }^{6}$. Andrew T. Marshall ${ }^{7,8} \cdot$ Krista M. Lisdahl $^{9}$. \\ Florence J. Breslin ${ }^{10}$. Amandine Van Rinsveld ${ }^{2}$. Sandra A. Brown ${ }^{1,11}$
}

Accepted: 21 December 2021 / Published online: 21 January 2022

(c) The Author(s), under exclusive licence to Springer Science+Business Media, LLC, part of Springer Nature 2022

\begin{abstract}
Parental knowledge/monitoring is negatively associated with adolescents' depressive symptoms, suggesting monitoring could be a target for prevention and treatment. However, no study has rigorously addressed the possibility that this association is spurious, leaving the clinical and etiological implications unclear. The goal of this study was to conduct a more rigorous test of whether knowledge/monitoring is causally related to depressive symptoms. 7940 youth (ages 10.5-15.6 years, 49\% female) at 21 sites across the U.S. completed measures of parental knowledge/monitoring and their own depressive symptoms at four waves 11-22 weeks apart during the COVID-19 pandemic. First, monitoring and depression were examined in standard, between-family regression models. Second, within-family changes in monitoring and depression between assessments were examined in first differenced regressions. Because the latter models control for stable, between-family differences, they comprise a stronger test of a causal relation. In standard, between-family models, parental monitoring and youths' depressive symptoms were negatively associated (standardized $\beta=-0.22,95 \% \mathrm{CI}=[-0.25,-0.20], p<0.001$ ). In first-differenced, within-family models, the association shrunk by about $55 \%$ (standardized $\beta=-0.10,95 \% \mathrm{CI}=[-0.12,-0.08], p<0.001$ ). The magnitude of within-family association remained similar when adjusting for potential time-varying confounders and did not vary significantly by youth sex, age, or history of depressive disorder. Thus, in this community-based sample, much of the prima facie association between parental knowledge/monitoring and youths' depressive symptoms was driven by confounding variables rather than a causal process. Given the evidence to date, a clinical focus on increasing parental knowledge/ monitoring should not be expected to produce meaningfully large improvements in youths' depression.
\end{abstract}

Keywords Adolescence $\cdot$ Depression $\cdot$ Parental monitoring

\section{Introduction}

Adolescent depression is common (Avenevoli et al., 2015), costly (Bodden et al., 2018), impairing (Asarnow et al., 2005), and associated with increased risk of poor long-term

William E. Pelham III

wpelham@ucsd.edu

1 Department of Psychiatry, University of California, San Diego, La Jolla, CA 92093, USA

2 Graduate School of Education, Stanford University, Palo Alto, CA 94305, USA

3 Department of Psychology, Florida International University, Miami, FL 33199, USA

4 Department of Psychiatry, University of Utah, Salt Lake City, UT 84108 , USA

5 Center for Health Sciences, SRI International, Menlo Park, CA 94025, USA outcomes (Clayborne et al., 2019). As such, there is strong interest in identifying modifiable protective factors that can prevent its occurrence. In a meta-analysis, Yap et al. (2014) identified one such protective factor: parental monitoring, defined as "structuring the child's home, school, and

6 Department of Psychology, Yale University, New Haven, CT 06520, USA

7 Department of Pediatrics, University of Southern California, Los Angeles, CA 90027, USA

8 Department of Pediatrics, Children's Hospital Los Angeles, Los Angeles, CA 90027, USA

9 Department of Psychology, University of Wisconsin at Milwaukee, Milwaukee, WI 53201, USA

10 National Center for Wellness \& Recovery, Oklahoma State University, Tulsa, OK 74136, USA

11 Department of Psychology, University of California, San Diego, La Jolla, CA 92093, USA 
community environments, and tracking the child's behavior in those environments" (Dishion \& McMahon, 1998, p. 66). 14 studies have linked parental knowledge/monitoring to adolescents' depression (pooled cross-sectional $r=-0.27$, pooled longitudinal $r=-0.14$ ). Yap and colleagues concluded that existing interventions targeting adolescent depression, which do not focus on improving parental monitoring, could be made more effective by incorporating monitoring-focused components borrowed from existing family-based interventions designed to reduce adolescent substance use or delinquency. The goal of the current study is to evaluate the extent to which parental monitoring is causally related to youths' depressive symptoms, thereby comprising a worthwhile target for intervention.

The studies included in Yap et al.'s (2014) meta-analysis were correlational, not experimental, so the apparent association between parental monitoring and adolescent depression could be due to confounding rather than a true causal relation. Indeed, monitoring and depression share many common antecedents. For example, parents monitor female adolescents more closely (Racz \& McMahon, 2011), and female adolescents are 2.3 times more likely than male adolescents to meet criteria for Major Depressive Disorder (MDD; Avenevoli et al., 2015). Thus, youth sex is a confounding variable, introducing statistical association between monitoring and depressive symptoms even in the absence of a causal relation. Shared genetics, socioeconomic status, marital status and conflict, stressful life events, parent-child relationship quality, parent psychopathology and alcohol/drug use, youth conduct problems, and community disadvantage may also confound this association (cf. Crouter \& Head, 2002; Racz et al., 2019; vs. Shore et al., 2018; Stirling et al., 2015). Credibly ruling out all these confounding factors is difficult.

The mechanisms that would explain a potential causal relation between parental monitoring and youths' depression are undertheorized. The parental monitoring construct was initially derived in the study of juvenile delinquency (Glueck \& Glueck, 1950, 1959), for which the theory is clear: Parents who better monitor their adolescents' whereabouts and activities have greater probability of discovering their delinquent behavior; most parents have rules prohibiting delinquent behavior and provide negative consequences for violations thereof; thus, youth who are more closely monitored are less likely to engage in delinquent behavior. In the context of depressive symptoms, it is less obvious why parental knowledge/monitoring of youths' whereabouts and activities would reduce youths' risk of depressive symptoms. Only one of the studies identified in Yap et al. (2014) contains any discussion of mechanisms potentially underlying the observed association. Hamza and Willoughby (2011) hypothesized that "parental attempts to solicit information from adolescents, and supervise adolescents' activities, communicate to adolescents that parents are interested in and concerned about their adolescents' well-being" (p. 903). Presumably, adolescents' perception of parents' interest in and concern about them in turn reduces their depressive symptoms.

Indeed, the role of parental monitoring is not explicitly addressed in prominent models of family processes in adolescent depression (Restifo \& Bögels, 2009; Sheeber et al., 2001). Monitoring may play a facilitative role to the betterestablished family processes which these models identify as protective against depression (e.g., parental support, teaching of affect regulation strategies). For example, parents who monitor youth more closely may be better aware of the occurrence of depressogenic events (e.g., breakup with romantic partner) or may be faster to notice early symptoms (e.g., social withdrawal, loss of interest in pleasurable activities), thus providing support or seeking evaluation/treatment more rapidly. However, such mechanisms are indirect and have not been explicated or tested in the published literature.

In summary, while many studies have linked parental knowledge/monitoring to youths' depressive symptoms, none has provided rigorous evidence of a causal relationship and the mechanisms that would explain such a relationship are undertheorized. Monitoring's role in a depression prevention or treatment program hinges on the assumption of a causal relationship-otherwise, improving parents' monitoring will not produce changes in youths' depressive symptoms. Thus, there is need for a more rigorous design that can determine the extent of the causal relation.

\section{Analysis of Within-Family Changes as Strategy to Improve Causal Inference}

To isolate the causal effect of monitoring on youths' depressive symptoms, we need a comparison of youth at different levels of monitoring yet the same levels on all factors that cause both monitoring and youths' depressive symptoms (i.e., confounding variables; Imbens \& Rubin, 2015). Measuring and adjusting for every way in which families with low, medium, and high monitoring differ would be difficult, owing to the large number of factors involved. A different way to address this problem is the use of withinperson analysis (Frijns et al., 2020). Rather than compare families that are low versus high in monitoring, we can instead compare the same youth's depressive symptoms under different levels of monitoring over time. The current study pursues such a within-family comparison using first differencing (Cameron \& Trivedi, 2005; Wooldridge, 2010), a regression-based technique that controls for unobserved, family-specific effects and thereby addresses a large class of potential confounding factors.

We conduct this within-family analysis using data collected during the COVID-19 pandemic, between May 2020 
and May 2021. The COVID-19 pandemic produced large and time-varying disruptions to families' daily lives and patterns of relation (Branje \& Morris, 2021; Glynn et al., 2021; Lebow, 2020; Liu et al., 2021), as many youth transitioned between in-person, hybrid, and remote schooling ( $\mathrm{MCH}$ Strategic Data, 2021); many parents transitioned between in-person and remote work (Brynjolfsson et al., 2020); stayat-home orders and social distancing recommendations were issued then rescinded (USA Today, 2021); and youths' contact with family and friends waxed and waned (CampioneBarr et al., 2021; Janssens et al., 2021; Rogers et al., 2021). Moreover, many studies have documented pandemic-related increases in depression, anxiety, and other mental health difficulties among adolescents (Racine et al., 2021; Singh et al., 2020). Thus, data collection during the pandemic may provide a unique opportunity for the analysis of within-family changes in monitoring and depression.

\section{Differentiating Multiple Aspects of the Knowledge/ Monitoring Construct}

It is also important to distinguish among multiple aspects of the monitoring construct (Dishion \& McMahon, 1998; Frijns et al., 2020; Guilamo-Ramos et al., 2010; Stattin \& Kerr, 2000). Measures of "parental monitoring" often collapse related but distinct parent-youth behaviors that may exhibit different impacts on youths' depression. Previous studies (Yap et al., 2014) have operationalized monitoring in multiple ways: parents' knowledge of youths' whereabouts and activities (e.g., Hamza \& Willoughby, 2011), parents' attempts to acquire knowledge (e.g., Sallinen et al., 2004), and the existence (Salem et al., 1998) and enforcement (Weaver \& Kim, 2008) of an evening curfew. Thus, while multiple aspects of the monitoring construct are associated with youths' depressive symptoms, it is unclear which are causally related.

\section{Heterogeneity of Effects}

Finally, it is important to explore how the protective effect of parental monitoring on youths' depressive symptoms varies across the population (Bergman \& Magnusson, 1997). This study focused on three potential sources of heterogeneity: youth sex, youth age, and youth history of depressive disorder. Two studies found stronger associations between monitoring and depression in females (Jacobson \& Crockett, 2000; Salem et al., 1998), while another did not (Kerr \& Stattin, 2000). No study has tested whether the association varies by youth age or history of depression. Monitoring exerts a stronger impact on externalizing outcomes among younger youth (Jacobson \& Crockett, 2000; Mak et al., 2020; Van Ryzin et al., 2012); the same may be true for depressive symptoms. Finally, if monitoring is important because it enables parental support, then parental knowledge of depressogenic events may be particularly important among youth with predisposition to depression (as indicated by diagnostic history) (Hazel et al., 2014).

\section{Current Study}

An emergent literature has linked parental knowledge/monitoring to adolescents' depressive symptoms. This study seeks to evaluate whether this link reflects (a) a causal relation, such that monitoring reduces depressive symptoms, or (b) a spurious association, introduced by confounding variables. We adjudicate between these possibilities using a longitudinal design that links within-family changes in parental knowledge/monitoring and adolescents' depressive symptoms during the COVID-19 pandemic, providing a stronger test of the causal relation.

\section{Method}

\section{Sample and Design}

Data were drawn from the Adolescent Brain and Cognitive Development $^{\text {SM }}$ (ABCD) Study. In 2016-2018, 11880 children ages 9-10 years old were recruited at 21 study sites across the United States (Garavan et al., 2018) and invited to complete biannual assessments thereafter. Recruitment was primarily school-based and exclusion criteria were minimal (e.g., youth did not speak English, MRI contraindications, major neurological or developmental disorder; Garavan et al., 2018). $48 \%$ of youth were female. Fifty two percent of youth were White, $15 \%$ were Black, $20 \%$ were Hispanic, and $11 \%$ identified as another racial/ethnic identity. Fiftyeight percent of youth had married parents/guardians, 56\% of youth had 1 + parent(s) with a bachelor's degree, and 39\% of youth lived in household were in households with annual income greater than $\$ 75,000$.

Data used in this study were collected between May 2020 and May 2021, during the coronavirus disease 2019 (COVID-19) pandemic. In May 2020, when youth were on average 12.4 years old $(S D=0.9$, range $=10.5-14.6)$, all participants in the ABCD Study ${ }^{\circledR}$ were invited to participate in a series of web-based surveys measuring the impact of the COVID-19 pandemic. All procedures were approved by the UCSD Human Research Protection Program-at the preceding assessment, parents had provided informed consent and permission for youth to participate and youth had provided informed assent. Legal guardians were sent links for a youth survey and a parent survey, each taking 10-15 min. The current study uses data from the four survey waves that measured youths' depressive symptoms, initiated on May 16, 2020 (Wave 1), August 4, 
2020 (Wave 2), December 13, 2020 (Wave 3), and May 17, 2021 (Wave 4). In total, 7,940 youth completed 19,608 surveys: 5,441 at Wave 1, 5,351 at Wave 2, 4,342 at Wave 3, and 4,474 at Wave 4. Survey waves were spaced 11-22 weeks apart. Compared to the full ABCD Study ${ }^{\circledR}$ sample, survey completers included fewer youth who were Black or whose parents had low education, low income, or were unmarried (Table S1). These differences were addressed through weighting, as described below.

\section{Parental Knowledge/Monitoring}

At all four waves, youth completed a 4-item measure of parental knowledge/monitoring (Chilcoat \& Anthony, 1996). Youth rated the following items on a 5-point Likert scale from never (0) to almost always (4), thinking of the past week: (1) "How often do your parents/guardians know where you are?", (2) "If you are at home when your parents or guardians are not, how often do you know how to get in touch with them?", (3) "How often do you talk to your mom/dad or guardian about your plans for the coming day, such as your plans about what will happen at school (or school-at-home) or what you are going to do?", and (4) "How many times do you and your parents/guardians eat dinner together?". This 4-item measure reflects the broad conceptualization of the monitoring construct that has been used in the vast majority of published literature on monitoring (Handschuh et al., 2020; Racz \& McMahon, 2011) and on the association between monitoring and depression in particular (Yap et al., 2014), tapping aspects including parent's knowledge of youth's whereabouts (Item 1), communication patterns when the youth is home alone (Item 2 ), youth disclosure and parent solicitation of information about youth's daily lives (Item 3 ), and parents' daily contact/involvement with youth (Item 4). As our measure captured multiple facets of monitoring, we (a) conducted psychometric analyses to verify a unidimensional scoring was appropriate (see supplement), (b) repeated all scale-level analyses separately for each item to ascertain potential differences, and (c) limited our conclusions to the knowledge/ monitoring construct broadly construed. Internal consistency was low (omega ranged from $0.50-0.55$ across Waves 1-4), as expected given the content breadth (Clifton, 2020). To improve measurement properties (McNeish \& Wolf, 2020), we fit an item response theory model and used estimated latent variable scores during analysis instead of simple sum scores (see supplement). The mean latent variable score equaled 0 at Wave $1(S D=0.70)$ and remained similar across Waves 2-4 (Table S2), with correlations between successive waves ranging $r=0.55-0.64$. Table $\mathrm{S} 2$ reports descriptive statistics for the latent variable scores, the scale items, and all other variables.

\section{Youths' Depressive Symptoms}

At all four waves, youth completed the Patient-Reported Outcomes Measurement Information System (PROMIS) pediatric short-form measure of depressive symptoms (v2.0) (Quinn et al., 2014). Youth rated eight items thinking of the past week: "I felt like I couldn't do anything right," "I felt everything in my life went wrong," "I felt unhappy," "I felt lonely," "I felt sad," "I felt alone," "I could not stop feeling sad," and "It was hard for me to have fun." Each item had five response options: never (0), almost never (1), sometimes (2), often (3) almost always (4). Internal consistency was very high (omega $=0.93-0.95$ across Waves $1-4$ ). For analysis, we computed the mean item response across the eight items. The mean was $0.85(S D=0.83)$ at Wave 1 , $0.78(S D=0.81)$ at Wave $2,0.95(S D=0.91)$ at Wave 3, and $0.94(S D=0.92)$ at Wave 4. Correlations between successive waves ranged $r=0.66-0.67$. The median change equaled 0 $(\mathrm{IQR}=[-0.38,0.25])$ between Wave 1 and Wave 2, 0.12 $(\mathrm{IQR}=[-0.12,0.50])$ between Wave 2 and Wave 3 , and 0 between Wave 3 and Wave $4(\mathrm{IQR}=[-0.38,0.25])$.

\section{History of Depressive Disorder}

Before the COVID-19 pandemic, parents completed a selfadministered, computerized, modified Kiddie Structured Assessment for Affective Disorders and Schizophrenia (KSADS; Kobak et al., 2020) at study entry and the 2-year follow-up visit (Barch et al., 2018). For each youth, we drew the most recently completed pre-pandemic assessment, which occurred a median of 15.9 months before the first survey during the COVID-19 pandemic. We then created an indicator of whether the youth met DSM-5 criteria for current or past Major Depressive Disorder, Persistent Depressive Disorder, or Unspecified Depressive Disorder-10.2\% of youth met this criterion. ${ }^{1}$

\section{Time-Varying Covariates}

The first differencing method controls for all time-invariant confounders, but it does not rule out time-varying confounders. Change in a third variable could cause change in parental monitoring and change in youths' depressive symptoms, producing a spurious association. We used the following procedure to address this issue. First, we reviewed the surveys, identifying any measured variable that might satisfy the criterion of $\Delta$ depression $_{i t} \leftarrow \Delta$ confounder $_{i t} \rightarrow \Delta$ monitoring $_{i t}$ and elaborating how these effects might occur. Second, we

\footnotetext{
${ }^{1}$ Impairment was not required for diagnosis of Major Depressive Disorder in ABCD 3.0 data release. All other DSM 5 criteria were required.
} 
brainstormed other potential time-varying confounders that were not measured on the surveys, drawing upon the published literature. We identified 14 time-varying covariates for inclusion-Table $S 3$ presents a justification of each selection and explains how they might serve as a confounder. Because it cannot be verified that a given variable is truly a confounder (Miller \& Chapman, 2001; Pearl, 2009), we took the approach of repeating each analysis while including/ excluding different sets of covariates in order to determine to what extent conclusions hinged on the specific covariates included. Potential confounders were grouped into sets as follows.

Youth health behaviors. Youth reported (a) whether they had used alcohol or drugs in the past 30 days (yes/no) (Lisdahl et al., 2018) and (b) how frequently they exercised or played outside in the past week (4-point Likert scale ranging from not at all to all of the time).

Parent emotionality factors. Parents rated (a) how much they were able to enjoy things in the past week (5-point Likert scale ranging from never to most of the time) and (b) how intensely they worried about COVID-19 in the past week (5-point Likert scale ranging from not at all to extremely) (NIH Intramural Research Program Mood Spectrum Collaboration, 2020).

Household events. Youth reported whether they were in school, either online or in-person. Parent reported whether (a) household was at increased risk of COVID due to work; (b) household engaged in social distancing, (c) avoided visiting others, or (d) avoided having visitors; (e) youth tested positive for COVID-19; (f) household experienced any material hardship in past month or (g) lost telephone service due to non-payment.

Family relations. Youth rated how the relationships between family members had been in the past week, ranging from $a$ lot worse to a lot better.

Separation. Youth reported whether they were temporarily separated from a close family member due to COVID-19.

\section{Analytic Plan}

Data were from the ABCD 4.0 data release (https://doi.org/ 10.15154/1523041), COVID-19 Survey First Data Release (https://doi.org/10.15154/1520584), and COVID-19 Survey Second Data Release (https://doi.org/10.15154/1522601). Analyses were conducted in R (R Core Team, 2021). Regressions were fit in the survey package (Lumley, 2003) using the identity link function. and clustering observations on study site, family, and youth. To address attrition, we estimated inverse probability weights that ensured completers of each survey wave were sociodemographically similar to the full ABCD Study ${ }^{\circledR}$ sample at study entry—all regression models were fit to the weighted data (see supplement). Both youth depressive symptoms and parental knowledge/monitoring were scaled by their standard deviation at the first survey wave.

Standard (between-family) regression models. We regressed youths' depressive symptoms on parental knowledge/monitoring, first in a univariate regression, then adding covariates for youth age, survey wave, and demographic variables. Next, we fit separate regressions that included a product term for the interaction of parental monitoring with youth sex, age, or history of depressive disorder, to test for moderation. Finally, we regressed youths' depressive symptoms on each monitoring scale item separately, to explore itemlevel differences.

First-differenced (within-family) regression models. There are several longitudinal models that could provide a more rigorous test of a causal relation than the standard regression models above (Grimm et al., 2016). Here, we use first differencing, which we view as providing a particularly rigorous test. Parental monitoring, depressive symptoms, and time-varying covariates were transformed to reflect change between consecutive waves, for each family (e.g., $\Delta$ monitoring $_{i, t}=$ monitoring $_{i, t}-$ monitoring $\left._{i, t-1}\right)$. Any factor that remained constant between two waves (e.g., youth sex, shared genetics, neighborhood disadvantage) could not explain covariation between the changes in monitoring and depressive symptoms and thus can no longer confound the association. Thus, regression models fit to the first differenced variables yield a more rigorous test of the causal relation than do standard regression models (Cameron \& Trivedi, 2005; Wooldridge, 2010). We fit an initial univariate regression of change in youth depressive symptoms on change in parental monitoring, then re-fit the model while including different sets of time-varying covariates thought to be potential confounders (as described above).

Sensitivity analysis for unmeasured confounding. We may not have included all confounders of the association between changes in monitoring and depressive symptoms. Per best practice (Liu et al., 2013), we conducted a sensitivity analysis to determine how strongly an unmeasured confounder would have to be correlated with change in monitoring and change in depressive symptoms in order to fully explain the observed association. If the required correlations are implausibly large, this would suggest that the association is robust against unmeasured confounding. 


\section{Results}

\section{Standard (Between-Family) Models}

Table 1 reports regressions of youths' depressive symptoms on parental knowledge/monitoring. In a univariate regression, parental knowledge/monitoring was negatively associated with youths' depressive symptoms (Coefficient [Coef.] $=-0.22$, Standard Error $[\mathrm{SE}]=0.01,95 \%$ $\mathrm{CI}=[-0.25,-0.20], p<0.001)$. A 1-standard-deviation increase in monitoring was associated with a 0.22 standard deviation decrease in youths' depressive symptoms. The estimated coefficient remained statistically significant and similar in magnitude when covarying survey wave; youth age, sex, and race/ethnicity; and parent education and marital status.

Moderation. The interaction of parental knowledge/monitoring and youth sex was statistically significant (coefficient on product term $=-0.14, \mathrm{SE}=0.03, p<0.001$ ); interactions with youth age $(p=1)$ and youth history of depressive disor$\operatorname{der}(p=0.59)$ were not (Table S4). The association between parental knowledge/monitoring and youths' depressive symptoms was significantly stronger among females $(\beta=-0.33)$ than males $(\beta=-0.18)$.

Item-by-item analysis. Table 1 reports item-by-item regressions. All four items from the parental monitoring scale were significantly associated with youths' depressive symptoms: Item $1(\beta=-0.27, \mathrm{SE}=0.03, p<0.001)$, Item $2(\beta=-0.13$, $\mathrm{SE}=0.02, p<0.001)$, Item $3(\beta=-0.14, \mathrm{SE}=0.01$, $p<0.001)$, and Item $4(\beta=-0.21, \mathrm{SE}=0.01, p<0.001)$. As with the scale-based analyses, coefficients relating each item to youths' depressive symptoms remained statistically significant and similar in magnitude when covarying survey wave; youth age, sex, and race/ethnicity; and parent education and marital status.

\section{First-Differenced (Within-Family) Models}

Figure 1 shows the estimated associations between parental knowledge/monitoring and youths' depressive symptoms in the standard models versus the first differenced

Table 1 Regression Models for Association Between Youth Depressive Symptoms and the Parental Knowledge/Monitoring Scale and Items

Independent Variable

\begin{tabular}{|c|c|c|c|c|c|}
\hline \multicolumn{6}{|c|}{ Dependent Variable: youths' depressive symptoms } \\
\hline $\begin{array}{l}\text { Model } \\
\text { Spec. } \\
1\end{array}$ & & $\begin{array}{l}\text { Model } \\
\text { Spec. } \\
2\end{array}$ & & $\begin{array}{l}\text { Model } \\
\text { Spec. } \\
\mathbf{3}\end{array}$ & \\
\hline $\begin{array}{l}\text { Coef } \\
\text { (SE) }\end{array}$ & $p$ & $\begin{array}{l}\text { Coef } \\
\text { (SE) }\end{array}$ & $p$ & $\begin{array}{l}\text { Coef } \\
\text { (SE) }\end{array}$ & $p$ \\
\hline $\begin{array}{l}-0.23 \\
(0.01)\end{array}$ & $<0.001$ & $\begin{array}{l}-0.22 \\
(0.01)\end{array}$ & $<0.001$ & $\begin{array}{l}-0.24 \\
(0.01)\end{array}$ & $<0.001$ \\
\hline $\begin{array}{l}-0.27 \\
(0.03)\end{array}$ & $<0.001$ & $\begin{array}{l}-0.27 \\
(0.03)\end{array}$ & $<0.001$ & $\begin{array}{l}-0.30 \\
(0.03)\end{array}$ & $<0.001$ \\
\hline $\begin{array}{l}-0.13 \\
(0.02)\end{array}$ & $<0.001$ & $\begin{array}{l}-0.14 \\
(0.02)\end{array}$ & $<0.001$ & $\begin{array}{l}-0.16 \\
(0.02)\end{array}$ & $<0.001$ \\
\hline $\begin{array}{l}-0.14 \\
(0.01)\end{array}$ & $<0.001$ & $\begin{array}{l}-0.14 \\
(0.01)\end{array}$ & $<0.001$ & $\begin{array}{l}-0.16 \\
(0.01)\end{array}$ & $<0.001$ \\
\hline $\begin{array}{l}-0.21 \\
(0.01)\end{array}$ & $<0.001$ & $\begin{array}{l}-0.20 \\
(0.01)\end{array}$ & $<0.001$ & $\begin{array}{l}-0.21 \\
(0.01)\end{array}$ & $<0.001$ \\
\hline 7,940 & & 7,940 & & 7,669 & \\
\hline 19,608 & & 19,608 & & 18,959 & \\
\hline No & & Yes & & Yes & \\
\hline No & & Yes & & Yes & \\
\hline No & & No & & Yes & \\
\hline
\end{tabular}

Parental knowledge/monitoring score

Item 1: How often do your parents/guardians know where you are?

Item 2: If you are at home when your parents or guardians are not, how often do you know how to get in touch with them?

Item 3: How often do you talk to your mom/dad or guardian about your plans for the coming day, such as your plans about what will happen at school (or school-at-home) or what you are going to do?

Item 4: How many times do you and your parents/guardians eat dinner together?

Number of youths

Number of observations

Covary youth age?

Covary survey wave?

Covary demographics: youth sex, youth race/ethnicity, parent education, parents married?

Three different model specifications (Model Spec. 1 - Model Spec. 3) were fit were fit for each independent variable. Specifications differed in which covariates were included: the bottom three rows of table indicate which covariates were included in each. The independent variables were examined in separate models (i.e., the knowledge/monitoring score and items were not covaried in the same regression). All models clustered on site, family, and youth. Parental knowledge/monitoring score was scaled by the standard deviation at Wave 1; item responses were scaled in original response metric (5-point Likert scale from never to almost always). Youths' depressive symptoms were scaled by the standard deviation at Wave 1 in all models

Coef coefficient, $S E$ standard error, $p$ p-value for test of statistical significance of coefficient 


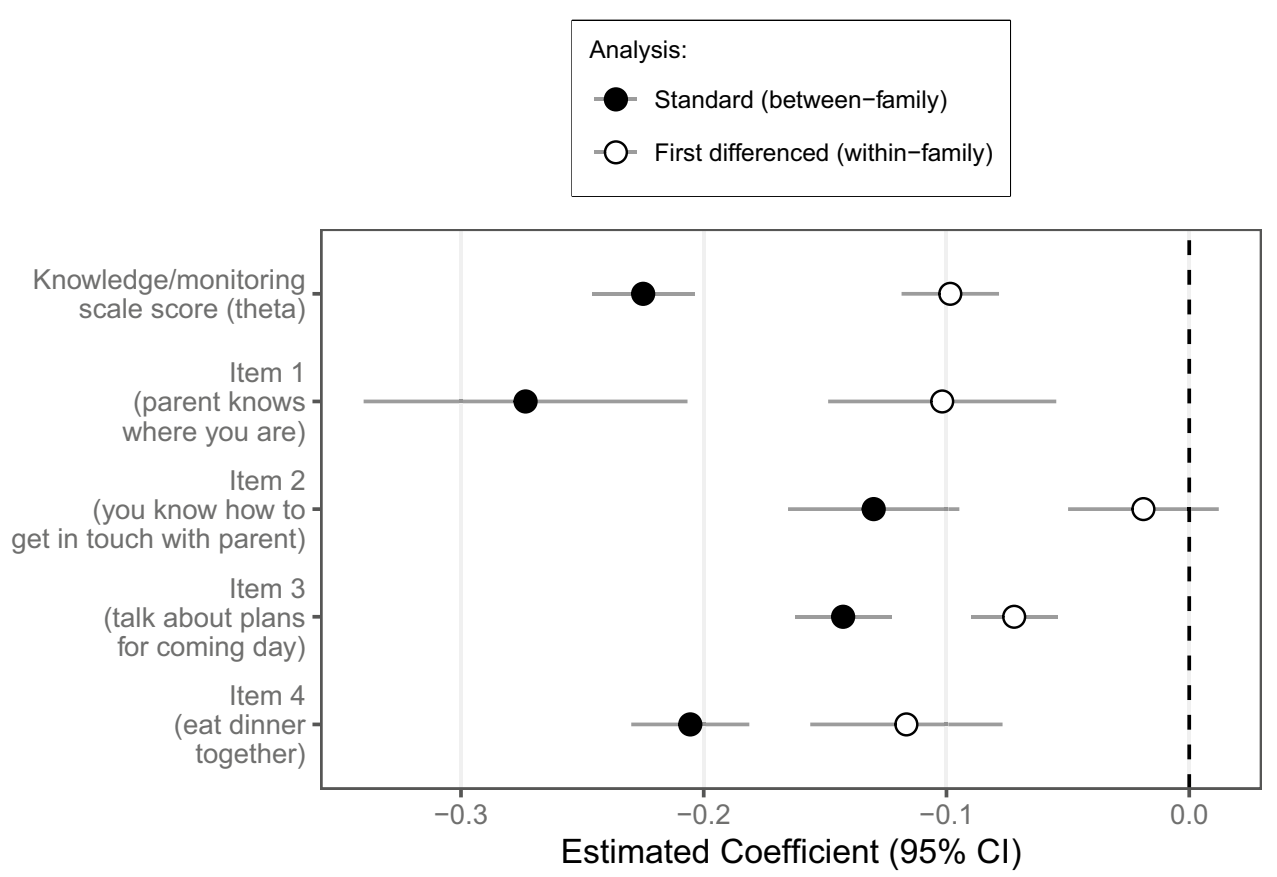

Fig. 1 Estimated Association between Parental Knowledge/Monitoring and Youths' Depressive Symptoms in Standard (Between-Family) Models vs. First Differenced (Within-Family) Models. Graphs the estimated coefficients from univariate regressions (Model Spec. 1 in Tables 1 and 2). Parental knowledge/monitoring scale score was scaled in standard deviation units; item responses were scaled in original response metric (5-point Likert scale from never to almost

models. Associations were substantially weaker in the first differenced models (Table 2). In a univariate regression, within-family changes in parental knowledge/monitoring were significantly associated with within-person changes in youths' depressive symptoms $(\beta=-0.10,95 \%$ $\mathrm{CI}=[-0.12,-0.08], p<0.001)$. A 1 -standard-deviation increase in monitoring was associated with a 0.10 standard deviation decrease in youths' depressive symptoms. The estimated coefficient remained similar in magnitude when covarying within-family changes in youth health behaviors, parent emotionality factors, household events, family relations, and/or separation from a close family member due to COVID-19 (Models 2-7).

Moderation. The interaction with within-family changes in parental knowledge/monitoring was not statistically significant for youth sex $(p=0.33)$, youth age $(p=0.62)$, or youth history of depressive disorder $(p=0.52)$ (Table S4).

Item-by-item analysis. Table 2 reports item-by-item regressions of first differences. In univariate regressions, the estimated coefficients were as follows: Item $1(\beta=-0.10$, SE $=0.02, p<0.001)$, Item $2(\beta=-0.02, \mathrm{SE}=0.02, p=0.25)$, Item $3(\beta=-0.07, \mathrm{SE}=0.01, p<0.001)$, and Item 4 always). Youths' depressive symptoms were scaled in standard deviation units in all models. As shown, the magnitude of association between parental knowledge/monitoring and youth depressive symptoms shrank substantially when moving from the standard models (black dots) to the first differenced models (white dots), at both the level of the scale and of the individual items

$(\beta=-0.12$, SE $0.02, p<0.001)$. The magnitude of each coefficient remained similar when covarying the time-varying covariates (Models 2-7).

\section{Sensitivity Analysis for Unmeasured Time-Varying Confounders}

We evaluated the vulnerability of the first differenced association between parental monitoring and youths' depressive symptoms ( $\beta=-0.10$, Table 2, Model 1) to unmeasured confounding. Let $r_{u x}$ and $r_{u y}$ be the correlation of a hypothetical unmeasured confounder with changes in youths' depressive symptoms and parental monitoring. See Fig. 2. Any combination of $r_{u x}$ and $r_{u y}$ that falls along the curve would attenuate the first differenced effect from $\beta=-0.10$ to $\beta=0$. For example, if there were an unmeasured confounder correlated at 0.31 with changes in monitoring and changes in youths' depressive symptoms (Point A in Fig. 2), then adjusting for this confounder would completely eliminate the association between monitoring and depressive symptoms. By convention (Cohen, 1988), this correlation is in the small-to-medium range. No single variable on the COVID surveys exhibited this level of correlation in change with parental monitoring (maximum observed value $=-0.10$ ). Thus, sensitivity analysis suggested the residual effect of $\beta=-0.10$ is at least somewhat robust to unmeasured confounding. 


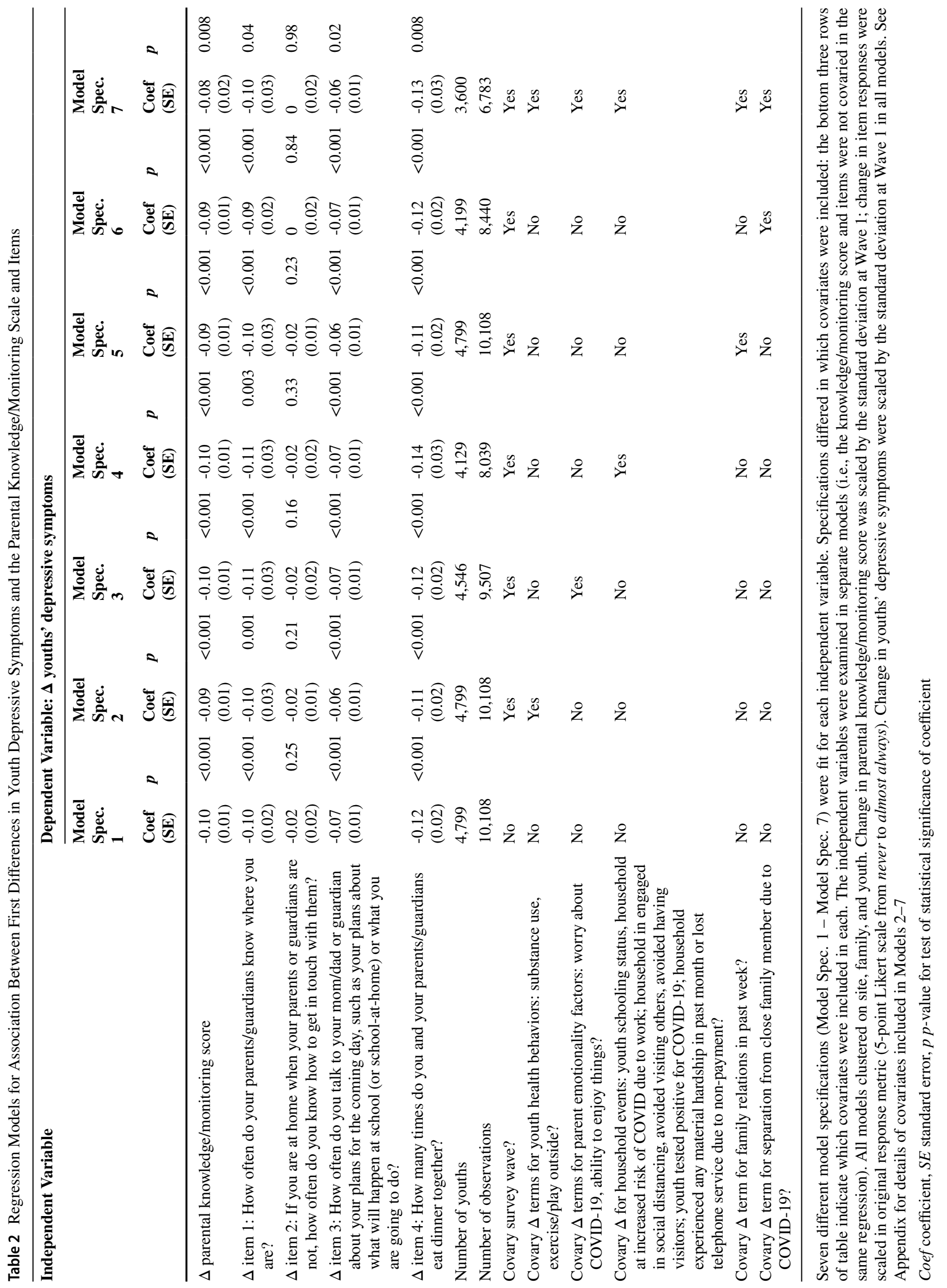




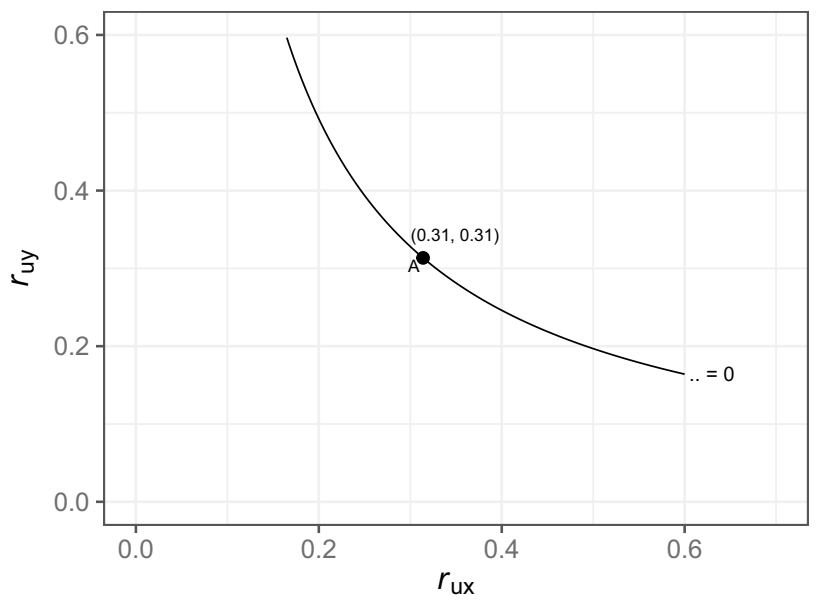

Fig. 2 Sensitivity Analysis for Unmeasured Time-Varying Confounder. $r_{u x}$ is the correlation between the unmeasured confounder and the change in parental knowledge/monitoring between survey waves. $r_{u y}$ is the correlation between the unmeasured confounder and the change in youths' depressive symptoms between survey waves. The estimated effect of parental knowledge/monitoring on youths' depressive symptoms was $\beta=-0.10$ (Table 2, Model Spec. 1). Points that fall along the curve show combinations of $r_{u x}$ and $r_{u y}$ that would attenuate that estimated effect of parental knowledge/monitoring from $\beta=-0.10$ to $\beta=0$. One such point is labeled (A) for reference: $r_{u x}=$ $0.31, r_{u y}=0.31$

\section{Discussion}

An emergent literature has documented that parental knowledge/monitoring is cross-sectionally and longitudinally associated with youths' depressive symptoms, motivating calls to adapt and modify existing prevention and intervention programs to incorporate a focus on increasing monitoring (Hamza \& Willoughby, 2011; Yap et al., 2014). However, the evidence supporting this association was correlational, not experimental, and the potential mechanisms explaining it were undertheorized. We conducted a more rigorous test of a causal relation using a longitudinal design in which 7,940 youth completed four waves of surveys approximately 11-22 weeks apart. In standard, between-family models, parental monitoring and youths' depressive symptoms were robustly associated. In first-differenced, within-family models, the association shrunk by about $55 \%$ and the effect size was very weak $(\beta=-0.10)$. Thus, findings contradict the hypothesis that parental knowledge/monitoring and youths' depressive symptoms have an average causal relation of clinically important magnitude.

\section{A Causal Protective Factor?}

Before addressing potential confounding factors, we replicated previous findings (Yap et al., 2014) in a large, nationwide, sociodemographically diverse sample: parental knowledge/monitoring and youth depressive symptoms were significantly associated ( $\beta=-0.22$ here, vs. $\beta=-0.27$ in Yap et al.). However, when addressing confounding via our first differenced regressions, the association of withinfamily changes in parents' monitoring and within-family changes in youths' depressive symptoms was very weak: a 1-SD increase in the monitoring measure was associated with a $0.10 S D$ decrease in youths' depressive symptoms. The narrow confidence interval $(95 \% \mathrm{CI}=[-0.12,-0.08])$ included only weak effect sizes. Clinical magnitude can be conceptualized by placing the estimated effect on the raw response metric. The depressive symptoms scale comprised eight items rated on 5-point Likert scales, with a total score ranging from 8 to $40 .-0.10 S D$ corresponds to a 0.66 unit reduction in the total scale score. Thus, a 1-standarddeviation increase in parental monitoring would be expected to reduce the item response for a single item by less than a single unit on the 5-point Likert scale, leaving the remaining seven item responses unchanged. A similarly weak effect size $(\beta=-0.05)$ was observed in the only published study that has adjusted for prior depressive symptoms when examining the monitoring-depression association (Hamza \& Willoughby, 2011). Thus, the evidence to date suggests that on average, parental monitoring is not an important etiological factor for youths' depressive symptoms in early to middle adolescence.

Moreover, the usefulness of targeting monitoring as a mechanism for clinical intervention to reduce depressive symptoms depends not only on monitoring's impact on depressive symptoms but on the intervention's ability to change monitoring. Intervention-induced increases in parents' monitoring of greater than one standard deviation are likely uncommon (e.g., Dishion \& Kavanagh, 2003; Henderson et al., 2009), so the expected effect of an intervention component targeting monitoring is likely weaker than $\beta=-0.10$. Thus, parental monitoring is unlikely to be a fruitful intervention target when trying to reduce depressive symptoms.

Findings for separate scale items were informative. In standard regressions, the item measuring parental knowledge (Item 1) had the strongest association with youth depressive symptoms ( $\beta=-0.27$ vs. $-0.13,-0.14$, and -0.21 ). However, the effect for the item measuring parental knowledge (Item 1) shrank the most in first differenced models, and it no longer had the strongest association $(\beta=-0.10$ vs. $-0.02,-0.07$, and -0.12 ). In fact, the strongest association in first differenced models was for the item measuring frequency of parent and youth dining together (Item 4), which hews furthest from the narrow conceptualization of monitoring as knowledge of youths' whereabouts and activities and likely captures additional aspects of parenting such as relationship quality and warmth (Kiss et al., in press). Thus, despite the pattern of prima facie associations, the 
more tangential aspects of the monitoring construct may explain a significant part of any causal effect of parental monitoring on youth depressive symptoms.

\section{Constraints on Generalizability}

Timescale of effects. We measured changes in parental monitoring and youths' depressive symptoms over approximately 11-22 weeks. There were clear reasons to expect an effect to manifest within this interval. Treatment effects on youths' depressive symptoms are detectable after six weeks in clinical trials (e.g., The TADS Team, 2007) and many evidence-based treatment protocols comprise 10-12 weeks of sessions (Weersing et al., 2017). Ample changes in parental monitoring occurred over the 11-22 weeks, with monitoring scores explaining a minority of variance (30-41\%) in monitoring scores at the subsequent wave. However, it remains possible that the effect of parental monitoring on youths' depressive symptoms operated over a more extended timescale than that examined in this study, perhaps taking many months or years to manifest. If this is the case, monitoring could be an important etiological factor while still not being a viable treatment target (given that it does not yield proximal change). Further evaluating and probing this possibility would require greater elaboration of the mechanisms that would explain such a delayed effect.

Non-clinical sample. Another possibility is that parental monitoring exerts a larger causal effect on depressive symptoms in youth at elevated risk of depression, with acute clinical depression, or with comorbid suicidal or self-injurious behavior. Regarding elevated risk, we found no evidence that the effect varied in youth who previously met criteria for depressive disorders. However, this was a community sample (as in all previous studies; Yap et al., 2014), so we could not evaluate monitoring's impact within the context of acute and severe clinical depression.

Data collection during the COVID-19 pandemic. Another possibility is that the very weak effect of parental monitoring is specific to the context of the COVID-19 pandemic, when these data were collected. We view this as unlikely. Our analyses and interpretation of findings hinged on the association between parental monitoring and depressive symptoms, so it is pandemic-related changes in the association (rather than the general level of monitoring or depressive symptoms) that would threaten the validity of findings. Thankfully, our pandemic data displayed an association between monitoring and depression similar in magnitude to the pre-pandemic studies included in Yap et al.'s (2014) meta-analysis ( $\beta=-0.22$ here vs. -0.27 in Yap et al.) and to pre-pandemic data from ABCD Study ${ }^{\circledR}$ participants $(\beta$
$=0.22$ here vs. -0.17 to -0.21 pre-pandemic).$^{2}$ Thus, the available data indicate the monitoring-depression association as studied in these pandemic data was representative of pre-pandemic conditions. Replication of findings outside the pandemic context is an important next step.

\section{Implications}

Clinical practice. Noting the prima facie association, some have called for a focus on monitoring to be incorporated into interventions for adolescent depression (Hamza \& Willoughby, 2011; Yap et al., 2014). Our findings of a very weak causal effect suggest that this focus would be misplaced and detract from finite intervention resources. Current treatment guidelines from the American Psychological Association (Weersing et al., 2017), American Academy of Pediatricians (Cheung et al., 2018), and American Academy of Child and Adolescent Psychiatrists (AACAP Work Group on Quality Issues, 2007) do not discuss or recommend targeting parental monitoring. This study supports that status quo.

Etiology. Findings suggest future investigations of the etiological role of monitoring in adolescent depression should use very large samples (given the likely weak effects). Elaborating and testing potential mechanisms that could underly a causal effect would help clarify whether the very weak effect we observed herein reflects monitoring playing a facilitative role to better-established risk/protective factors (e.g., more monitoring enables more timely social support) or perhaps is due to residual confounding. These investigations should consider differences in mechanisms that could explain a greater impact of monitoring among female adolescents, as was observed in our data.

\section{Strengths and Limitations}

Strengths of this study include the large and sociodemographically diverse sample; the prospective, longitudinal design; and the use of first differencing to strengthen causal inference. Limitations identified above include the non-clinical nature of the sample and that data were collected entirely during the COVID-19 pandemic. In addition, youth were in early to middle adolescence. The broader literature on monitoring suggests that its impact grows weaker as youth enter later adolescence (Mak et al.,

\footnotetext{
${ }^{2}$ For comparison, we calculated the correlation between the same parental knowledge/monitoring scale and youth report of three depressive symptoms on the Brief Problem Monitor (Achenbach, 2009) at ABCD Study ${ }^{\circledR}$ waves completed before the pandemic. The correlation ranged from -0.17 to -0.21 across waves.
} 
2020; Racz \& McMahon, 2011), so the absence of participants in late adolescence would not seem to explain the weak effects observed herein. Finally, our brief, broadband measure of parental knowledge/monitoring did not distinguish all facets of the construct, such as knowledge arising from youth disclosure vs. parent solicitation (Stattin \& Kerr, 2000). Future work should measure and investigate multiple facets of the monitoring construct to better tease apart their potential independent relations with youths' depressive symptoms.

\section{Conclusion}

If there is a causal relation between parental knowledge/ monitoring and adolescents' depressive symptoms, it may be very weak on average. Much of the prima facie association between these constructs was due to confounding variables. A single study is insufficient to prove or disprove causality (Ohlsson \& Kendler, 2019) and our findings should be replicated outside the pandemic context. However, taking our findings in conjunction with previous work (Yap et al., 2014), at present, there is little evidence supporting the belief that increasing parents' monitoring will produce clinically meaningful reductions in adolescents' depressive symptoms. For now, clinicians should focus on intervention targets with a better-established relationship to depression.

Supplementary Information The online version contains supplementary material available at https://doi.org/10.1007/s10802-021-00896-9.

Author Contributions Pelham contributed to the design and execution of the study protocol, collaborated in conceptualization of analyses and planning of the manuscript, conducted the analyses, and wrote the first draft of the manuscript. Tapert contributed to the design and execution of the study protocol, collaborated in conceptualization of analyses and planning of the manuscript, and edited the manuscript for critically important intellectual content. Gonzalez, Guillaume, Dick, Sheth, Baker, Baskin-Sommers, Marshall, Lisdahl, Breslin, and Van Rinsveld contributed to the design and execution of the study protocol and edited the manuscript for critically important intellectual content. Brown contributed to the design and execution of the study protocol, collaborated in conceptualization of analyses and planning of the manuscript, and edited the manuscript for critically important intellectual content.

Funding Data used in the preparation of this article were obtained from the Adolescent Brain Cognitive Development ${ }^{\mathrm{SM}}$ (ABCD) Study (https:// abcdstudy.org), held in the NIMH Data Archive (NDA). This is a multisite, longitudinal study designed to recruit more than 10000 children age 9-10 and follow them over 10 years into early adulthood. The ABCD Study ${ }^{\circledR}$ is supported by the National Institutes of Health and additional federal partners under award numbers U01DA041048, U01DA050989, U01DA051016, U01DA041022, U01DA051018, U01DA051037, U01DA050987, U01DA041174, U01DA041106, U01DA041117, U01DA041028, U01DA041134, U01DA050988, U01DA051039, U01DA041156, U01DA041025, U01DA041120, U01DA051038, U01DA041148, U01DA041093, U01DA041089, U24DA041123, and U24DA041147. A full list of supporters is available athttps://abcdstudy. org/federal-partners.html. Additional support for this work was made possible from supplements to U24DA041123 and U24DA041147, the National Science Foundation (NSF 2028680), and Children and Screens: Institute of Digital Media and Child Development Inc. A listing of participating sites and a complete listing of the study investigators can be found at https://abcdstudy.org/Consortium_Members.pdf. ABCD consortium investigators designed and implemented the study and/or provided data but did not necessarily participate in analysis or writing of this report. This manuscript reflects the views of the authors and may not reflect the opinions or views of the $\mathrm{NIH}$ or $\mathrm{ABCD}$ consortium investigators.

Availability of Data and Material The $\mathrm{ABCD}$ data repository grows and changes over time. The $\mathrm{ABCD}$ data used in this report came from the ABCD 4.0 data release (https://doi.org/10.15154/1523041), the ABCD COVID-19 Survey First Data Release (https://doi.org/10.15154/1520584), and the ABCD COVID-19 Survey Second Data Release (https://doi.org/ 10.15154/1522601). A single construct-youth's history of depressive disorders-was drawn from the ABCD 3.0 data release (https://doi.org/ 10.15154/1519007). DOIs can be found at https://nda.nih.gov/study.html? id=901, https://nda.nih.gov/study.html?id=1299, https://nda.nih.gov/ study.html?\&id=1041, and https://nda.nih.gov/study.html?\&id=1225.

\section{Compliance with Ethical Standards}

Ethical Approval The authors assert that all procedures contributing to this work comply with the ethical standards of the relevant national and institutional committees on human experimentation and with the Helsinki Declaration of 1975, as revised in 2008. All procedures were approved by the UCSD Human Research Protection Program (Protocol \#160091).

Informed Consent At the ABCD Study ${ }^{\circledR}$ assessment completed immediately preceding the pandemic, parents provided informed consent and permission for youth to participate and youth provided informed assent.

Conflicts of Interest The authors have no relevant financial or nonfinancial interests to disclose.

\section{References}

AACAP Work Group on Quality Issues. (2007). Practice parameter for the assessment and treatment of children and adolescents with depressive disorders. Journal of the American Academy of Child \& Adolescent Psychiatry, 46, 1503-1526.

Achenbach, T. M. (2009). The Achenbach System of Empirically Based Assessment (ASEBA): Development Findings Theory and Applications. University of Vermont Research Center for Children, Youth, and Families.

Asarnow, J. R., Jaycox, L. H., Duan, N., LaBorde, A. P., Rea, M. M., Tang, L., \& Wells, K. B. (2005). Depression and role impairment among adolescents in primary care clinics. Journal of Adolescent Health, 37, 477-483.

Avenevoli, S., Swendsen, J., He, J.-P., Burstein, M., \& Merikangas, K. R. (2015). Major depression in the National Comorbidity Survey-Adolescent Supplement: Prevalence correlates and treatment. Journal of the American Academy of Child \& Adolescent Psychiatry, 54, 37-44.

Barch, D. M., Albaugh, M. D., Avenevoli, S., Chang, L., Clark, D. B., Glantz, M. D., \& Sher, K. J. (2018). Demographic, physical and mental health assessments in the adolescent brain and cognitive development study: Rationale and description. Developmental Cognitive Neuroscience, 32, 55-66. 
Bergman, L. R., \& Magnusson, D. (1997). A person-oriented approach in research on developmental psychopathology. Development and Psychopathology, 9, 291-319.

Bodden, D. H. M., Stikkelbroek, Y., \& Dirksen, C. D. (2018). Societal burden of adolescent depression, an overview and cost-of-illness study. Journal of Affective Disorders, 241, 256-262.

Branje, S., \& Morris, A. S. (2021). The impact of the COVID-19 pandemic on adolescent emotional, social, and academic adjustment. Journal of Research on Adolescence, 31, 486-499.

Brynjolfsson, E., Horton, J. J., Ozimek, A., Rock, D., Sharma, G., \& TuYe, H.-Y. (2020). COVID-19 and remote work: An early look at us data (No. w27344). National Bureau of Economic Research.

Cameron, A. C., \& Trivedi, P. K. (2005). Microeconometrics: Methods and Applications. Cambridge University Press.

Campione-Barr, N., Rote, W., Killoren, S. E., \& Rose, A. J. (2021). Adolescent adjustment during COVID-19: The role of close relationships and COVID-19-related stress. Journal of Research on Adolescence, 31, 608-622.

Cheung, A. H., Zuckerbrot, R. A., Jensen, P. S., Laraque, D., Stein, R. E. K., \& Group, G.-P. S. (2018). Guidelines for adolescent depression in primary care (GLAD-PC): Part II Treatment and ongoing management. Pediatrics. https://doi.org/10.1542/peds.2017-4082

Chilcoat, H. D., \& Anthony, J. C. (1996). Impact of parent monitoring on initiation of drug use through late childhood. Journal of the American Academy of Child \& Adolescent Psychiatry, 35, 91-100.

Clayborne, Z. M., Varin, M., \& Colman, I. (2019). Systematic review and meta-analysis: Adolescent depression and long-term psychosocial outcomes. Journal of the American Academy of Child \& Adolescent Psychiatry, 58, 72-79.

Clifton, J. D. W. (2020). Managing validity versus reliability trade-offs in scale-building decisions. Psychological Methods, 25, 259-270.

Cohen, J. (1988). Statistical Power Analysis for the Behavioral Sciences (2nd ed.). Lawrence Erlbaum Associates.

Crouter, A. C., \& Head, M. R. (2002). Parental monitoring and knowledge of children. In M. H. Bornstein (Ed.), Handbook of Parenting (1st ed., Vol. 3, pp. 461-483). Lawrence Erlbaum Associates.

Dishion, T. J., \& Kavanagh, K. (2003). Intervening in Adolescent Problem Behavior: A Family-Centered Approach. Guilford Press.

Dishion, T. J., \& McMahon, R. J. (1998). Parental monitoring and the prevention of child and adolescent problem behavior: A conceptual and empirical formulation. Clinical Child and Family Psychology Review, 1, 61-75.

Frijns, T., Keijsers, L., \& Finkenauer, C. (2020). Keeping secrets from parents: On galloping horses, prancing ponies and pink unicorns. Current Opinion in Psychology, 31, 49-54.

Garavan, H., Bartsch, H., Conway, K., Decastro, A., Goldstein, R. Z., Heeringa, S., \& Zahs, D. (2018). Recruiting the ABCD sample: Design considerations and procedures. Developmental Cognitive Neuroscience, 32, 16-22.

Glueck, S., \& Glueck, E. (1950). Unraveling Juvenile Delinquency. New York, NY: The Commonwealth Fund.

Glueck, S., \& Glueck, E. (1959). Predicting Delinquency and Crime. Harvard University Press.

Glynn, L. M., Davis, E. P., Luby, J. L., Baram, T. Z., \& Sandman, C. A. (2021). A predictable home environment may protect child mental health during the COVID-19 pandemic. Neurobiology of Stress, 14, 100291.

Grimm, K. J., Ram, N., \& Estabrook, R. (2016). Growth Modeling: Structural Equation and Multilevel Modeling Approaches. Guilford Publications.

Guilamo-Ramos, V., Jacquard, J., \& Dittos, P. (Eds.). (2010). Expert perspectives on parental monitoring. In Parental Monitoring of Adolescents: Current Perspectives for Researchers and Practitioners (pp. 205-266). New York, NY: Columbia University Press.
Hamza, C. A., \& Willoughby, T. (2011). Perceived parental monitoring, adolescent disclosure, and adolescent depressive symptoms: A longitudinal examination. Journal of Youth and Adolescence, 40, 902-915.

Handschuh, C., Mokkink, L. B., \& Smaldone, A. (2020). Perceived parental monitoring: A systematic review of monitoring instruments. Journal of Nursing Measurement, 28, E253-E292.

Hazel, N. A., Oppenheimer, C. W., Technow, J. R., Young, J. F., \& Hankin, B. L. (2014). Parent relationship quality buffers against the effect of peer stressors on depressive symptoms from middle childhood to adolescence. Developmental Psychology, 50, 2115-2123.

Henderson, C. E., Rowe, C. L., Dakof, G. A., Hawes, S. W., \& Liddle, H. A. (2009). Parenting practices as mediators of treatment effects in an early-intervention trial of multidimensional family therapy. The American Journal of Drug and Alcohol Abuse, 35, 220-226.

Imbens, G. W., \& Rubin, D. B. (2015). Causal Inference in Statistics, Social, and Biomedical Sciences. Cambridge University Press.

Jacobson, K. C., \& Crockett, L. J. (2000). Parental monitoring and adolescent adjustment: An ecological perspective. Journal of Research on Adolescence, 10, 65-97.

Janssens, J. J., Achterhof, R., Lafit, G., Bamps, E., Hagemann, N., Hiekkaranta, A. P., \& Kirtley, O. J. (2021). The impact of COVID19 on adolescents' daily lives: The role of parent-child relationship quality. Journal of Research on Adolescence, 31, 623-644.

Kerr, M., \& Stattin, H. (2000). What parents know, how they know it, and several forms of adolescent adjustment: Further support for a reinterpretation of monitoring. Developmental Psychology, $36,366-380$.

Kobak, K., Townsend, L., Birmaher, B., Milham, M., \& Kaufman, J. (2020). Computer-assisted psychiatric diagnosis. Journal of the American Academy of Child \& Adolescent Psychiatry, 59, $213-215$

Lebow, J. L. (2020). Family in the age of COVID-19. Family Process. https://doi.org/10.1111/famp.12543

Lisdahl, K. M., Sher, K. J., Conway, K. P., Gonzalez, R., Feldstein Ewing, S. W., Nixon, S. J., \& Heitzeg, M. (2018). Adolescent brain cognitive development (ABCD) study: Overview of substance use assessment methods. Developmental Cognitive Neuroscience, 32, 80-96.

Liu, J., Zhou, T., Yuan, M., Ren, H., et al. (2021). Daily routines parent-child conflict and psychological maladjustment among Chinese children and adolescents during the COVID-19 pandemic. Journal of Family Psychology. https://doi.org/10.1037/ fam0000914

Liu, W., Kuramoto, S. J., \& Stuart, E. A. (2013). An introduction to sensitivity analysis for unobserved confounding in nonexperimental prevention research. Prevention Science, 14, 570-580.

Lumley, T. (2003). Analysis of complex survey samples. Journal of Statistical Software, 9, 1-19.

Mak, H. W., Russell, M. A., Lanza, S. T., Feinberg, M. E., Fosco, G. M., \& Fosco, G. M. (2020). Age-varying associations of parental knowledge and antisocial peer behavior with adolescent substance use. Developmental Psychology, 56, 298-311.

MCH Strategic Data. (2021). COVID-19 Impact: School District Status-Updates for Spring 2021. Retrieved February 11, 2021, from https://www.mchdata.com/covid19/schoolclosings

McNeish, D., \& Wolf, M. G. (2020). Thinking twice about sum scores. Behavior Research Methods. https://doi.org/10.3758/ s13428-020-01398-0

Miller, G. A., \& Chapman, J. P. (2001). Misunderstanding analysis of covariance. Journal of Abnormal Psychology, 110, 40-48.

NIH Intramural Research Program Mood Spectrum Collaboration. (2020). The CoRonavIruS Health Impact Survey (CRISIS) V0.2 Adult SelfReport Baseline Form: Short Form. Bethesda, MD. Retrieved 
from https://www.phenxtoolkit.org/toolkit_content/PDF/CRISIS_ Baseline_Adult_Life_Changes.pdf

Ohlsson, H., \& Kendler, K. S. (2019). Applying causal inference methods in psychiatric epidemiology: A review. JAMA Psychiatry. https://doi.org/10.1001/jamapsychiatry.2019.3758

Pearl, J. (2009). Causal inference in statistics: An overview. Statistics Surveys, 3, 96-146.

Quinn, H., Thissen, D., Liu, Y., Magnus, B., Lai, J.-S., Amtmann, D., \& DeWalt, D. A. (2014). Using item response theory to enrich and expand the PROMIS ${ }^{\circledR}$ pediatric self report banks. Health and Quality of Life Outcomes, 12, 160.

R Core Team. (2021). $R$ : A language and environment for statistical computing. Vienna, Austria: R Foundation for Statistical Computing.

Racine, N., McArthur, B. A., Cooke, J. E., Eirich, R., Zhu, J., \& Madigan, S. (2021). Global prevalence of depressive and anxiety symptoms in children and adolescents during COVID-19: A meta-analysis. JAMA Pediatrics. https://doi.org/10.1001/ jamapediatrics.2021.2482

Racz, S. J., \& McMahon, R. J. (2011). The relationship between parental knowledge and monitoring and child and adolescent conduct problems: A 10-year update. Clinical Child and Family Psychology Review, 14, 377-398.

Racz, S. J., McMahon, R. J., King, K. M., Pinderhughes, E. E., \& Bendezú, J. J. (2019). Kindergarten antecedents of the developmental course of active and passive parental monitoring strategies during middle childhood and adolescence. Development and Psychopathology, 31, 1675-1694.

Restifo, K., \& Bögels, S. (2009). Family processes in the development of youth depression: Translating the evidence to treatment. Clinical Psychology Review, 29, 294-316.

Rogers, A. A., Ha, T., \& Ockey, S. (2021). Adolescents' perceived socioemotional impact of COVID-19 and implications for mental health: Results from a U.S.-based mixed-methods study. Journal of Adolescent Health, 68, 43-52.

Salem, D. A., Zimmerman, M. A., \& Notaro, P. C. (1998). Effects of family structure family process and father involvement on psychosocial outcomes among African American adolescents. Family Relations, 47, 331-341.

Sallinen, M., Kinnunen, U., \& Rönkä, A. (2004). Adolescents' experiences of parental employment and parenting: Connections to adolescents' well-being. Journal of Adolescence, 27, 221-237.

Sheeber, L., Hops, H., \& Davis, B. (2001). Family processes in adolescent depression. Clinical Child and Family Psychology Review, 4, 19-35.
Shore, L., Toumbourou, J. W., Lewis, A. J., \& Kremer, P. (2018). Review: Longitudinal trajectories of child and adolescent depressive symptoms and their predictors - a systematic review and meta-analysis. Child and Adolescent Mental Health, 23, 107-120.

Singh, S., Roy, D., Sinha, K., Parveen, S., Sharma, G., \& Joshi, G. (2020). Impact of COVID-19 and lockdown on mental health of children and adolescents: A narrative review with recommendations. Psychiatry Research, 293, 113429.

Stattin, H., \& Kerr, M. (2000). Parental monitoring: A reinterpretation. Child Development, 71, 1072-1085.

Stirling, K., Toumbourou, J. W., \& Rowland, B. (2015). Community factors influencing child and adolescent depression: A systematic review and meta-analysis. Australian \& New Zealand Journal of Psychiatry, 49, 869-886.

The TADS Team. (2007). The Treatment for Adolescents With Depression Study (TADS): Long-term effectiveness and safety outcomes. Archives of General Psychiatry, 64, 1132-1143.

USA Today. (2021). COVID-19 restrictions: Map of COVID-19 case trends, restrictions, and mobility. Retrieved February 11, 2021, from https://www.usatoday.com/storytelling/coronavirusreopening-america-map/

Van Ryzin, M. J., Fosco, G. M., \& Dishion, T. J. (2012). Family and peer predictors of substance use from early adolescence to early adulthood: An 11-year prospective analysis. Addictive Behaviors, 37, 1314-1324.

Weaver, S. R., \& Kim, S. Y. (2008). A person-centered approach to studying the linkages among parent-child differences in cultural orientation, supportive parenting, and adolescent depressive symptoms in Chinese American families. Journal of Youth and Adolescence, 1, 36-49.

Weersing, V. R., Jeffreys, M., Do, M.-C.T., Schwartz, K. T. G., \& Bolano, C. (2017). Evidence base update of psychosocial treatments for child and adolescent depression. Journal of Clinical Child \& Adolescent Psychology, 46, 11-43.

Wooldridge, J. M. (2010). Econometric Analysis of Cross Section and Panel Data. MIT Press.

Yap, M. B. H., Pilkington, P. D., Ryan, S. M., \& Jorm, A. F. (2014). Parental factors associated with depression and anxiety in young people: A systematic review and meta-analysis. Journal of Affective Disorders, 156, 8-23.

Publisher's Note Springer Nature remains neutral with regard to jurisdictional claims in published maps and institutional affiliations. 